\title{
Probing Complex Nanostructures by Combining Atomic-Scale Theory and Scanning Transmission Electron Microscopy
}

\author{
Sokrates T. Pantelides ${ }^{1,2}$ and Stephen J. Pennycook ${ }^{3}$ \\ 1. Department of Physics and Astronomy, Vanderbilt University, Nashville, TN 37235 USA \\ 2. Materials Science and Technology Division, Oak Ridge National Laboratory, Oak Ridge, TN 37831 \\ USA \\ 3. Department of Materials Science and Engineering, National University of Singapore, Singapore \\ 117576
}

Aberration-corrected scanning transmission electron microscopy has reached extraordinary levels of spatial and energy resolution, in both imaging and electron-energy-loss spectroscopy (EELS). In parallel, calculations based on density functional theory (DFT) using high-performance computers have made enormous strides in describing the atomic-scale properties of complex materials. A combination of theory and microscopy is a powerful approach to resolve long-standing issues and unveil new phenomena. This talk focuses on select recent applications of the combined approach.

The first example is the elucidation of the origin of white light emission by ultrasmall CdSe nanoparticles [1]. Proposed explanations invoked surface and defect states, but could not account for the observed continuous spectrum. Atomic-resolution Z-contrast images found that nanoparticles larger than $2 \mathrm{~nm}$ in diameter have a solid crystalline core, but sub-2-nm particles are fluxional under the electron beam. Noting that, under uv excitation, energy is transferred to vibrations before a band-gap photon is emitted, quantum dynamical simulations at the corresponding temperature found that a sub-2-nm particle is similarly fluxional. These fluctuations cause the band gaps to vary continuously across the visual range on a femtosecond time scale (Fig. 1c), leading to white light (Fig. 1b).

The second example is the resolution of the long-standing puzzle of the origin of ferromagnetism in insulating $\mathrm{LaCoO}_{3}$ thin films grown epitaxially under strain [2]. The insulating nature of the films led to the assumption that the films are stoichiometric (oxygen vacancies would make the material n-type). Several attempts using first-principles calculations could not reproduce the experimental data. Columnresolved EELS (Fig. 2) established that observed dark stripes in Z-contrast images and EELS maps are caused by ordered vacancies. DFT calculations found that such structures are indeed ferromagnetic. Finally, it was found that just insertion of the oxygen vacancies without relaxation introduces only Peierls-like minigaps within the metallic band structure. Relaxation of the structure, however, leads to a rupture of the energy bands and the appearance of a large energy gap, as observed (Fig. 2).

The third example is the discovery of a new crystalline order in $\mathrm{CuInS}_{2}$ nanoparticles, called interlaced crystals [3]. In $\mathrm{CuInS}_{2}, \mathrm{Cu}$ and In atoms share one of two sublattices, but XRD cannot resolve whether the cations are ordered. Valence-balancing electron counting suggests that $\mathrm{Cu}$ and In cations should be ordered. DFT calculations indeed found that several ordering possibilities induce no strain at all and have essentially identical energies. Z-contrast images found that the different ordering possibilities coexist on a totally undisturbed perfect Bravais lattice (Fig. 3). Calculations found that domain and phase boundaries, which are not uniquely assigned, cost no energy. These are the features of what has been labeled as interlaced crystalline order. Such materials should be ideal for thermoelectric applications as the boundaries have negligible effect on electronic conductivity but reduced thermal conductivity. 
Finally, a new scheme that combines DFT with diffraction theory to simulate probe-position-dependent EELS will be described briefly [4].

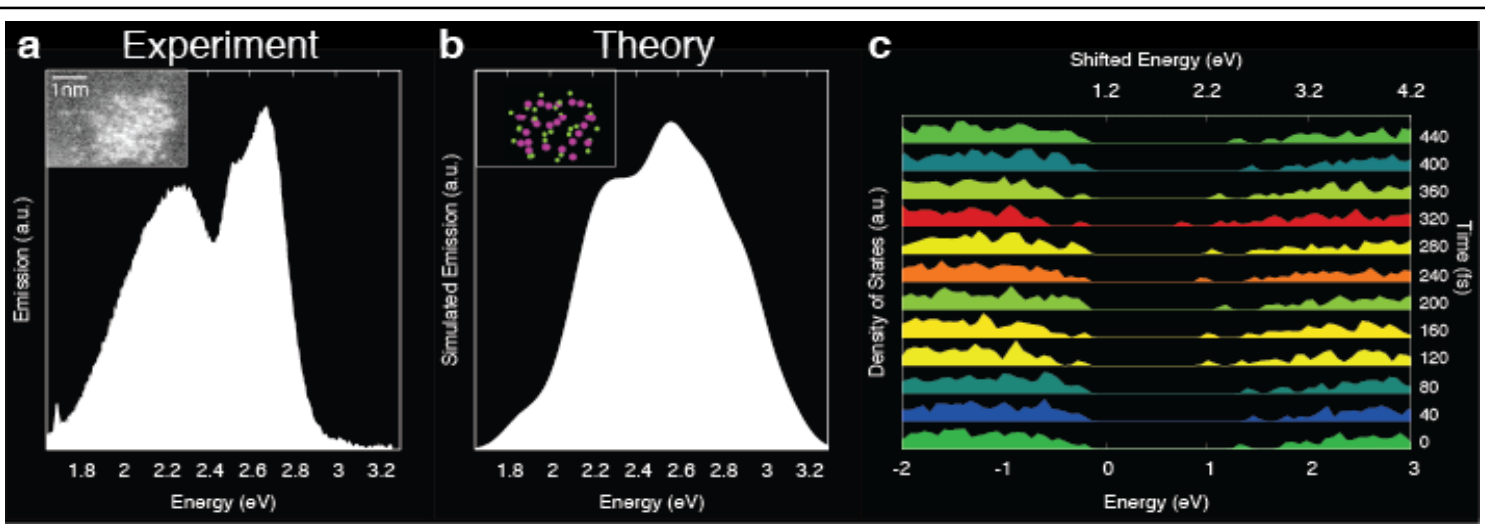

Figure 1. (a-b) Experimental and simulated white light spectrum of sub-2-nm CdSe nanoparticles shown in the insets (c) Fluctuating band gap in the density of states produces the continuous spectrum. From Ref. [1].
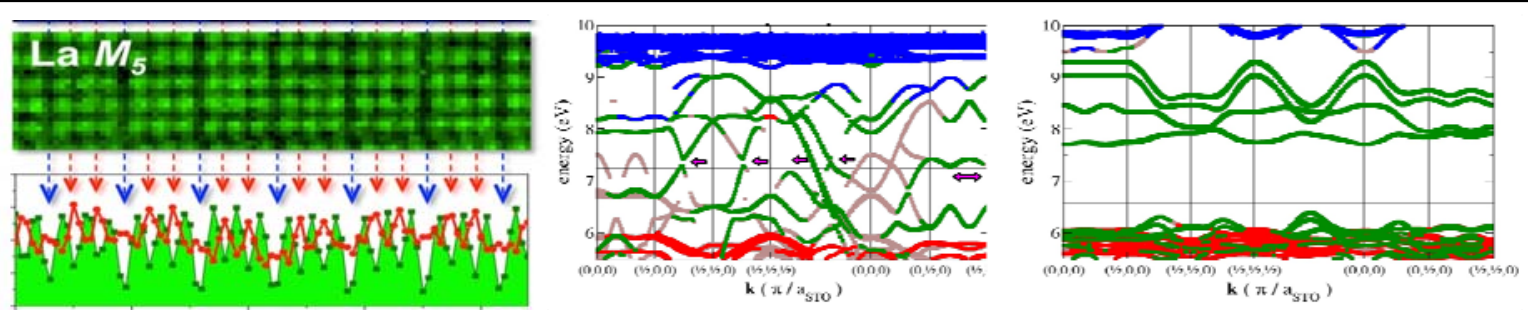

Figure 2. Left: La M $\mathrm{M}_{5}$ EELS map and O K EELS (shaded in green), showing O depletion in the dark stripes. Right: LCO spin-up bands after ordered vacancies are inserted, and after relaxation. Ref. [2]

\section{References:}

[1] T. J. Pennycook, J. R. McBride, S. J. Rosenthal, S. J. Pennycook, and S. T. Pantelides, "Dynamic fluctuations in ultrasmall nanocrystals induce white light emissions", Nano Lett. 12, 3038-3042 (2012).

[2] N. Biskup, J. Salafranca, V. Mehta, M. P. Oxley, Y. Suzuki, S. J. Pennycook, S. T. Pantelides, and M. Varela, "Insulating Ferromagnetic $\mathrm{LaCoO}_{3-\delta}$ films: A phase induced by ordering of oxygen vacancies", Phys. Rev. Lett. 112, 087202 (2014).

[3] X. Shen, E. A. Hernández-Pagan, W. Zhou, Y. S.

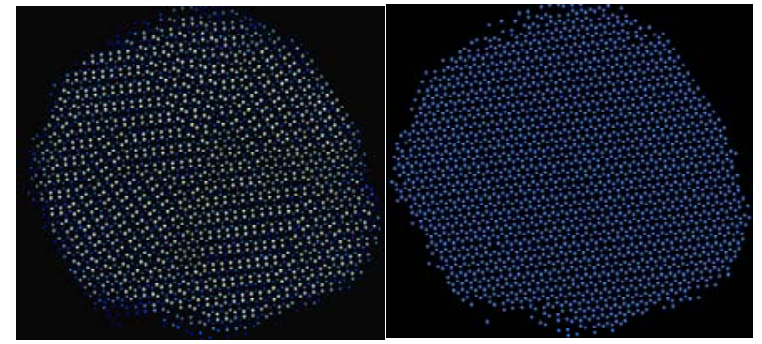

Figure 3. Z-contrast image of $\mathrm{CuInS}_{2}$ nanoparticle and the same image using the same color for both $\mathrm{Cu}$ and In atoms, revealing a perfect Bravais lattice. Ref. [3].

Puzyrev, J.-C. Idrobo, J. E. Macdonald, S. J. Pennycook, and S. T. Pantelides, "Interlaced crystals having a perfect Bravais lattice and complex chemical order revealed by real-space crystallography", Nature Commun. 5, 5431 (2014).

[4] Collaborators in this work are identified in the cited references. Research at Vanderbilt was supported by Department of Energy grant DE-FG02-09ER46554. Microscopy was carried out at Oak Ridge National Laboratory, supported by the Department of Energy, Office of Science, Basic Energy Sciences, Materials Science and Engineering Division. Computations were carried out at the National Energy Research Scientific Computing Center, also supported by the Department of Energy. 